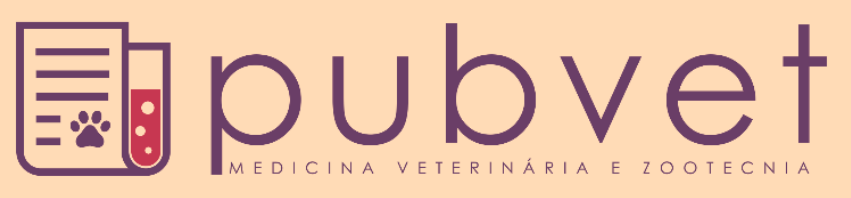

ISSN $1982-1263$

https://doi.org/10.31533/pubvet.v14n1a500.1-9

\title{
Sistema de aquaponia em escada: estudo de caso
}

\author{
Ana Lucia de Oliveira Belintano ${ }^{10}$, Franciele Itati Kreutz ${ }^{10}$, Eliseu Aparecido Messias ${ }^{10}$, \\ Josias Rodrigues Ibanhez $^{1}$, Milena Wolff Ferreira ${ }^{2 * \bullet}$, Denilson de Oliveira Guilherme ${ }^{3 \bullet}$
}

${ }^{1}$ Acadêmica da Universidade Católica Dom Bosco, Curso de Zootecnia, Campo Grande, MS Brasil.

${ }^{2}$ Professora da Universidade Católica Dom Bosco, Curso de Zootecnia, Campo Grande, MS Brasil.

${ }^{3}$ Professor da Universidade Católica Dom Bosco, Curso de Agronomia, Campo Grande, MS Brasil.

*Autor para correspondência,E-mail: milenawolff@ucdb.br

Resumo. O crescente aumento da população mundial e a necessidade de suprir toda demanda nutricional, exige o desenvolvimento de novas técnicas de produção alimentar. Assim surge a aquaponia, que integra o cultivo de peixes e a agricultura hidropônica, promovendo um sistema sustentável de produção de alimentos seguros e conservação dos recursos hídricos. O sistema baseia-se na recirculação de água entre os cultivos, utilizando substratos da produção dos peixes para o crescimento da hortaliça evitando o acúmulo da amônia tóxica excretada pelos animais na água, viabilizando a conversão aeróbia através da ação dos microrganismos, consequentemente absorvidos pelas raízes das plantas auxiliando seu crescimento. Este trabalho acompanhou a qualidade de água e o desempenho produtivo de tilápias do Nilo (Oreochromis niloticus) criadas em sistema aquapônico em escada, consorciada com Alface-Crespa (Lactuca sativa var. crispa). Os resultados foram comparados com os recomendados pela literatura e superaram a expectativa de crescimento e ganho de peso dos peixes com biomassa inicial de $1.500 \mathrm{~g} / \mathrm{m}^{3}$ e biomassa final de $9.856 \mathrm{~g} /$ $\mathrm{m}^{3}$, tendo o peso médio inicial de $50 \mathrm{~g}$ e final de $352 \mathrm{~g}$, com consumo médio de ração $945 \mathrm{~g} /$ peixe e 2,68 de conversão alimentar. Observou-se que os parâmetros de qualidade de água durante o período de início e multiplicação da ação das bactérias nitrificadoras de amônia apresentaram variações, que podem ter relação com o percentual de $11,12 \%$ de mortalidade dos alevinos. Após este período os resultados obtidos foram satisfatórios e corresponderam ao esperado comprovando que o sistema de aquaponia em escadas apresenta diversos benefícios e requer conhecimento técnico para resultados satisfatórios, sendo uma alternativa viável de cultivo e manejo da aquaponia no Brasil.

Palavras chave: água, amônia, recirculação, tilápia

\section{Aquaponics ladder system: case study}

Abstract. The growing increase in the world population and the need to meet all nutritional demand requires the development of new food production techniques. Thus, comes aquaponia, which integrates fish cultivation and hydroponic agriculture, promoting a sustainable system of producing safe food and conservation of water resources. The system is based on the recirculation of water between crops, using fish production substrates for vegetable growth, avoiding the accumulation of toxic ammonia excreted by animals in water, enabling aerobic conversion through the action of microorganisms, consequently absorbed by plant roots, helping their growth. This work accompanied the quality of water and the productive performance of Nile tilapia (Oreochromis niloticus) created in an aquaponic system in stairs, intercropmed with Lettuce (Lactuca sativa var. crispa). The results were compared with those recommended in the literature and exceeded the expectation of growth and weight gain of fish with initial biomass of $1,500 \mathrm{~g} / \mathrm{m}^{3}$ and final biomass of $9,856 \mathrm{~g} / \mathrm{m}^{3}$, with the initial average weight of $50 \mathrm{~g}$ and final weight of $352 \mathrm{~g}$, with 
average feed intake $945 \mathrm{~g} /$ fish and 2.68 feed. It was observed that the parameters of water quality during the period of beginning and multiplication of the action of ammonia nitrir bacteria presented variations, which may be related to the percentage of $11.12 \%$ of fingerling mortality. After this period the results obtained were satisfactory and corresponded to the expected proving that the aquaponicsystem on stairs presents several benefits and requires technical knowledge for satisfactory results, being a viable alternative for cultivation and management of aquaponics in Brazil.esumo em inglês. A palavra abstract em maiúsculo e negrito.

Keywords: water, ammonia, recirculation, tilapia

\section{Sistema de escalera de acuaponía: estudio de caso}

Resumen. El creciente aumento de la población mundial y la necesidad de satisfacer toda la demanda nutricional requiere el desarrollo de nuevas técnicas de producción de alimentos. Así viene la aquaponia, que integra el cultivo de peces y la agricultura hidroponica, promoviendo un sistema sostenible de producción de alimentos seguros y conservación de los recursos hídricos. El sistema se basa en la recirculación del agua entre cultivos, utilizando sustratos de producción de pescado para el crecimiento vegetal, evitando la acumulación de amoníaco tóxico excretado por los animales en el agua, permitiendo la conversión aeróbica a través de la acción de microorganismos, consecuentemente absorbidos por las raíces de las plantas, ayudando a su crecimiento. Este trabajo acompañó la calidad del agua y el rendimiento productivo de la tilapia del Nilo (Oreochromis niloticus) criadas en un sistema acuapípónico en cascadas, asociada con Lechuga-Crespa (Lactuca sativa var. crispa). Los resultados se compararon con los recomendados en la literatura y superaron la expectativa de crecimiento y aumento de peso de los peces con biomasa inicial de $1.500 \mathrm{~g} / \mathrm{m}^{3}$ y biomasa final de $9.856 \mathrm{~g} / \mathrm{m}^{3}$, con el peso medio inicial de $50 \mathrm{~g}$ y el peso final de $352 \mathrm{~g}$, con una ingesta promedio de concentrado $945 \mathrm{~g} /$ pescado y 2,68 de conversión alimentar. Se observó que los parámetros de la calidad del agua durante el período de inicio y multiplicación de la acción de las bacterias nitrificadoras de amoníaco presentaban variaciones, que pueden estar relacionadas con el porcentaje del $11,12 \%$ de la mortalidad de alevinos. Después de este período los resultados obtenidos fueron satisfactorios y correspondieron a lo esperado demostrando que el sistema de aquaponía en cascada presenta varios beneficios y requiere conocimientos técnicos para resultados satisfactorios, siendo una alternativa viable para el cultivo y manejo de la acuaponía en Brasil.

Palabras clave: agua, amoníaco, recirculación, tilapia

\section{Introdução}

No Brasil a produção de peixes cultivados cresce a cada ano, segundo o Anuário Peixe BR de 2019, a produção aquícola brasileira cresceu 4,5\% e atingiu 722.560 toneladas. Entre as espécies de água doce, a tilápia representa $55,7 \%$ da produção, apresentando crescimento de 11,9\% em 2018. Além disso, a produção de 357.639 toneladas de tilápias em 2018 proporcionou ao Brasil se manter como $4^{\circ}$ maior produtor de tilápia no cenário mundial (IBGE, 2019).

Grande parte da produção nacional de peixes é realizada em viveiros de terra caracterizando sistema semi-intensivo de produção, em que é realizada a fertilização orgânica para aumentar a produção primária e fornecimento de alimento natural aos peixes, além da suplementação com ração (Castagnolli, 1992; Souza \& Teixeira Filho, 1985) e de acordo com Crepaldi et al. (2006), este é o sistema mais antigo de produção de peixes. Assim, a capacidade de expansão e sustentabilidade da piscicultura depende do aumento de produtividade ou aumento de áreas produtoras.

O desenvolvimento sustentável de uma produção é baseado em três componentes: a produção lucrativa, a preservação do meio ambiente e o desenvolvimento social (Eler \& Millani, 2007). Assim, para que a produção seja consolidada, é preciso que as condições ambientais sejam respeitadas e atendidos os parâmetros de qualidade de água especificados pela legislação brasileira. Também é preciso 
que a qualidade dos efluentes gerados nas pisciculturas seja o menor possível, a fim de que as alterações provocadas nos corpos hídricos à jusante sejam minimizadas.

Dessa forma, a aquaponia surge como uma alternativa de intensificação da produção de maneira sustentável. Os desenhos de um sistema de aquaponia podem variar. No entanto, de acordo com Rakocy (2007) é necessário à realização de três processos complementares, o cultivo dos peixes no tanque onde há a entrada de nutrientes na forma de ração, a nitrificação das diferentes formas de apresentação do nitrogênio em filtros biológicos e mesas de hidroponia que fazem a absorção de nutrientes pela parcela vegetal do sistema.

Neste sentido objetivou-se com este trabalho descrever o desenvolvimento de um sistema intensivo de produção de tilápias no modelo de aquaponia em escadas.

\section{Relato de caso}

Este trabalho encontra-se registrado e aprovado pela Comissão de Ética no Uso de Animais (CEUA) da Universidade Católica Dom Bosco sob o protocolo $n^{\circ}$ 019/2017. Estando de acordo com os preceitos da Lei $n^{\circ} 11.794$, de outubro de 2008, do Decreto ${ }^{\circ}$ 6.899, de 15 de julho de 2009, e com as normas editadas pelo Conselho Nacional de Controle de Experimentação Animal (CONCEA).

A aquaponia é um sistema de água recirculando onde, em comparação com o sistema de recirculação convencional (RAS) que incide apenas na produção de peixes, a unidade de biofiltro para nitrificação é redefinida por uma unidade hidropônica (Love et al., 2014; Rakocy, 2012) e pode ser localizado indoor ou ao ar livre. Além da utilização dos nutrientes dos resíduos dos peixes reciclados para o crescimento das plantas no sistema hidropônico, reduzindo os impactos negativos ao ambiente. Kubitza (2006) que os componentes básicos de um sistema de aquaponia são: tanque de cultivo de peixes, decantadores e filtros, biofiltros, sistema de aeração/oxigenação, sistema de bombas e tubulações de distribuição e retorno da água.

Dessa forma, a instalação de sistemas de aquaponia tem como principal limitante o alto custo inicial de implantação e alta demanda por energia elétrica para o funcionamento do sistema de bombas e aeração. $\mathrm{O}$ sistema de aquaponia mesmo sendo de pequeno tamanho, pode ser considerado como investimento inicialmente mais caro (Rakocy, 2007; Rakocy et al., 2016). Entretanto, o sistema em escada prevê a simplificação do filtro biológico e a diminuição do uso de energia elétrica para um único bombeamento de água do tanque de cultivo dos peixes para o filtro biológico e, em seguida a água passa do filtro biológico para as caixas de cultivo de vegetais por gravidade (escada), e o retorno da água para o tanque de cultivo de peixes gera uma movimentação de água que é responsável pela aeração, dispensando o sistema de aeradores. Além disso, o sistema de aquaponia em escadas montados neste trabalho em casa de vegetação não requer aquecedores para a manutenção da temperatura da água.

Três estruturas de aquaponia em escadas foram montadas para possuir componentes uniformes alocados em casa de vegetação de $50 \mathrm{~m}^{2}$. Cada sistema foi composto de uma caixa de cultivo de peixes, com capacidade total de água de $1.000 \mathrm{~L}$ acoplada ao filtro biológico e três degraus de escadas, e cada degrau apresenta 4 bandejas de cultivo de vegetais, conforme ilustrado na Figura 1.

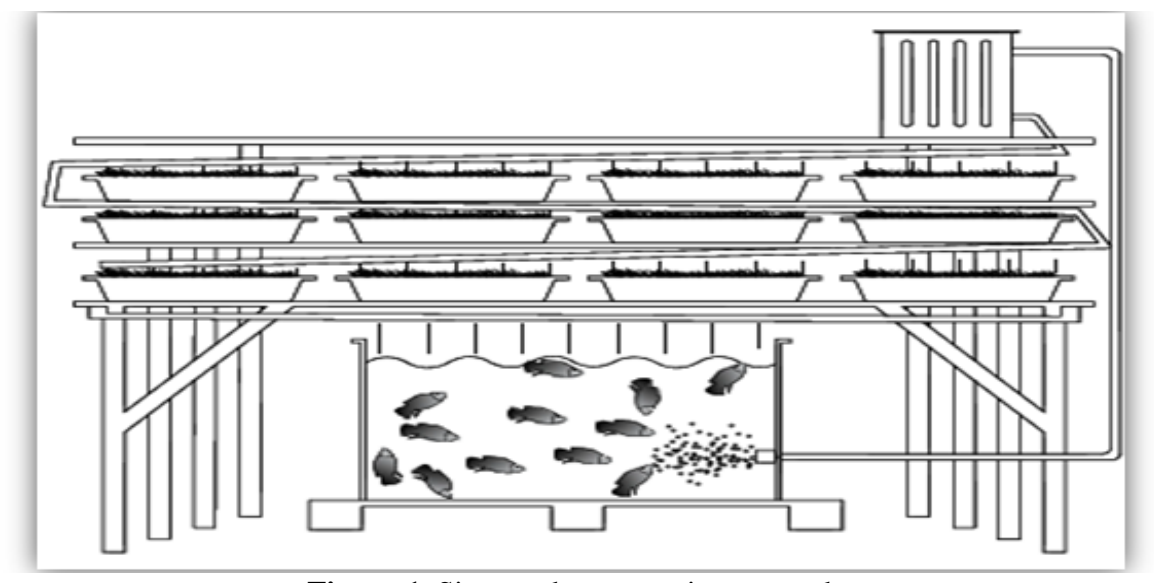

Figura 1. Sistema de aquaponia em escada. 
As caixas d'água com capacidade total de $1.000 \mathrm{~L}$ foram utilizadas para o cultivo de tilápias. Em cada caixa foram alocados 30 alevinos com peso médio de $50 \mathrm{~g}$, totalizando uma biomassa inicial de 1,5 $\mathrm{kg} / \mathrm{m}^{3}$. A biomassa máxima esperada era de $15 \mathrm{~kg} / \mathrm{m}^{3}$. As tilápias foram alimentadas com ração comercial para onívoros em crescimento, $0,4 \mathrm{~mm}$, contendo $32 \%$ de $\mathrm{PB}$, calculados para atender $3 \%$ da biomassa do tanque, e ajustada a cada biometria, divididos em duas alimentações diárias.

Para a determinação do desempenho das tilápias foi medido peso inicial, biomassa inicial, peso final. Biomassa final, sobrevivência, ganho de peso médio diário, consumo médio de ração e conversão alimentar aparente.

No sistema de aquaponia em escada a água é bombeada do tanque de cultivo de peixes para a parte superior do filtro biológico, em uma altura de $2,5 \mathrm{~m}$, por meio de moto bombas periféricas $0,5 \mathrm{CV}$ monofásica QB 60220 volts. O filtro biológico é composto, por um tambor de 50L de capacidade, preenchidos com 2 metros de tela e $15 \mathrm{~L}$ de cacos de cerâmica. A filtragem mecânica se dá pela passagem de água na tela, com o intuito de reter resíduos sólidos nesse compartimento. Esse sistema de filtragem mecânica na tela tem a finalidade de simplificar os filtros de decantação descritos em sistemas de recirculação (Maucieri et al., 2018).

Em seguida a nitrificação da amônia tem início pelas bactérias nitrificadoras aderidas à cerâmica. A filtragem biológica, nesse sistema, é caracterizada pela ação das bactérias que serão responsáveis por transformar a amônia tóxica, excretada pelos peixes, em nitrito e depois nitrato, que é uma substância absorvida pelas plantas (Junge et al., 2017).

Cada bandeja de cultivo de vegetais apresenta dimensões de $0,3 \times 0,5 \mathrm{~m}$, sendo $0,15 \mathrm{~m}^{2}$ por bandeja, totalizando $1,8 \mathrm{~m}^{2}$ de área de cultivo de vegetais em cada estrutura. Dessa forma, a razão $\mathrm{m}^{2} \mathrm{de}$ hidroponia $/ \mathrm{m}^{3}$ de tanque de peixes nessa estrutura foi de 1,8. Danaher et al. (2013) descreveram razão hidroponia/tanque de peixes $\left(\mathrm{m}^{2} / \mathrm{m}^{3}\right)$ de 1,9 , em sistema de hidroponia flutuante e criação de tilápias. Razões menores de 1,4 e 1,2 foram relatadas por Kloas et al. (2015) e Castillo-Castellanos et al. (2016), respectivamente, ambos trabalhando com tilápias.

As caixas de cultivo de vegetais apresentam capacidade total de $20 \mathrm{~L}$ que foi preenchido com $10 \mathrm{~L}$ argila expandida, pois de acordo com Jordan et al. (2018) são excelentes substrato para os vegetais, assim como para as bactérias nitrificadoras, além de ser mais leve e resistente. Argila expandida favorece a entrada de ar na cama de cultivo, é mais porosa favorecendo então o desenvolvimento das colônias de bactérias, a argila expandida, por sua vez contribui com a redução das temperaturas, é muito usado em hidroponia por ter uma capacidade boa de drenagem, permite a sustentação das plantas, conformação regular, resulta em melhor aeração e menor retenção de umidade (Sátiro et al., 2018).

Espécies e variedades vegetais adaptadas a hidroponia são sempre recomendadas para a aquaponia, uma vez que a maioria delas têm o crescimento ótimo entre o $\mathrm{pH}$ de 5,8 e 6,2, toleram altos teores de água em suas raízes e significativas variações nos teores de nutrientes dissolvidos na solução nutritiva, sem apresentar sintomas de deficiência nutricional (Hundley \& Navarro, 2013). Dessas, foram utilizadas duas mudas de alface da variedade crespa em cada bandeja de cultivo. A alface foi semeada em bandejas de polietileno expandido com 288 células, E após apresentarem seu segundo par de folhas foram transplantadas para o sistema de aquaponia previamente em funcionamento com os peixes por volta de 20 dias.

As características limnológicas da água foram mensuradas semanalmente. A temperatura e o oxigênio dissolvido foram mensurados com o auxílio de um oxímetro digital (Alfakit AT-160), para o pH utilizou-se peagâmetro digital portátil (Akso AK-95), e a amônia foi medida por kit colorimétrico Labcon ${ }^{\circledR}$.

\section{Discussão}

A produção em sistema de aquaponia em escadas foi acompanhada por três meses. Nesse período a temperatura média foi de 28,17 , sendo a máxima registrada de 32 , e a mínima de $25,3^{\circ} \mathrm{C}$. O oxigênio dissolvido variou de 5,1 a $6,5 \mathrm{mg} / \mathrm{L}$. O menor valor observado de $\mathrm{pH}$ foi de 6,4 e o maior valor 7,14 . A média de amônia foi de 1,37 ppm, e a média de nitrato foi $0,76 \mathrm{ppm}$ (Gráfico 1).

Os peixes dependem diretamente da temperatura da água para manter a sua temperatura corporal e seu metabolismo funcionando corretamente. $\mathrm{O}$ metabolismo do peixe é maior à medida que aumenta $\mathrm{a}$ 
temperatura da água, mas isso até um limite de temperatura. Se a temperatura for superior a $32^{\circ} \mathrm{C}$ pode ocorrer mortalidade; abaixo de $24^{\circ} \mathrm{C}$ decresce o apetite rapidamente e aumenta a chance de proliferação de doenças (Garcia et al., 2008). Segundo El-Sayed (2006), quando a temperatura da água diminui, as tilápias reduzem o consumo, diminuem o ritmo biológico e, consequentemente, o desempenho piora.nE, devido às condições controladas da casa de vegetação onde a aquaponia estava instalada a temperatura se manteve dentro do conforto térmico das tilápias de 27 a $30^{\circ}$ o que garante uma boa taxa de crescimento (Somerville et al., 2014).

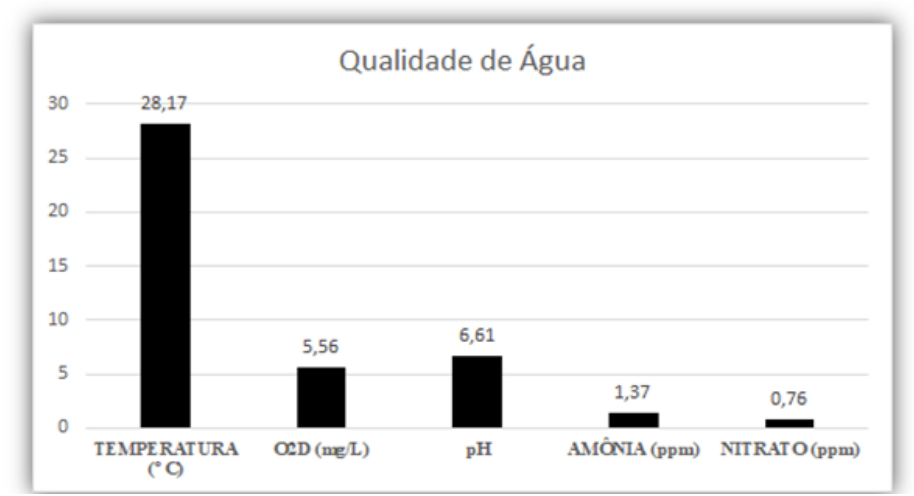

Gráfico 1. Médias de qualidade de água durante o período de criação.

Em sistemas de recirculação e sistemas que não possuem fitoplânctons para a produção de oxigênio pela fotossíntese a fonte de oxigênio são compressores de ar, na sua maioria, que demandam energia elétrica e podem onerar o custo de produção e, em função dessa característica Love et al. (2014) descrevem o alto consumo de energia elétrica como grande desvantagem do sistema de aquaponia.

No sistema em escada do presente trabalho, a concentração de oxigênio da água foi garantida pela movimentação da água ao retornar das caixas de vegetação para as caixas de cultivo de peixes, produzindo bolhas que incorporaram o oxigênio atmosférico a água. Durante todo o tempo de acompanhamento, o oxigênio dissolvido apresentou concentração adequada (Boyd, 2000). A tilápia apresenta baixa exigência em oxigênio dissolvido, vivendo perfeitamente em águas contendo até 1,2 $\mathrm{mg} / \mathrm{L}$ (Mercante et al., 2004). As concentrações de oxigênio dissolvido no sistema apresentaram máxima de $6,5 \mathrm{mg} / \mathrm{L}$ e mínima $5,1 \mathrm{mg} / \mathrm{L}$, que está dentro da faixa considerada ideal para a criação de tilápias.

$\mathrm{O}$ valor de $\mathrm{pH}$ é bastante importante do sistema de aquaponia pois além de influenciar o crescimento dos peixes (Garcia et al., 2008) influencia ainda na disponibilidade de nutrientes para as plantas (Tyson et al., 2008). Nutrientes como Fe, Mn, B, Zn e Cu decresce drasticamente em disponibilidade em níveis de $\mathrm{pH}$ superiores a 7,0; nutrientes como $\mathrm{Mg}, \mathrm{P}, \mathrm{Ca}$ e molibdênio (Mo) decrescem em solubilidade em níveis de pH inferiores a 6,0 (Domingues et al., 2012). Nesse sistema foi observado pH médio de 5,56 e uma pequena oscilação ao longo da criação. Dessa forma, se manteve na faixa adequada a produção de tilápias e próximo aos valores de pH descritos por Kloas et al. (2015). Sátiro et al. (2018) mencionam que o pH também tem relação com o ciclo de nitrificação do nitrogênio, sendo esse o principal elemento do sistema que é excretado pelos peixes na forma de amônia, mineralizado pelas bactérias e absorvido pelos vegetais no sistema de aquaponia.

As bactérias nitrificantes dos gêneros Nitrosomonas e Nitrobacter de ocorrência natural e responsáveis pela nitrificação do amoníaco, são predominantemente aeróbias e têm $\mathrm{pH}$ ótimo entre 7,0 e 8,0 (Pereira \& Mercante, 2005). A partir da segunda semana de criação os níveis de amônia começaram a aumentar, chegando ao valor máximo de $3,8 \mathrm{ppm}$ na quarta semana. Após a quarta semana foi observado a queda gradual na quantidade de amônia até estabilizar em $0,25 \mathrm{ppm}$ na décima segunda semana. A quantidade de nitrato permaneceu em zero até a quarta semana quando apresentou $0,25 \mathrm{ppm}$. Atingindo valor máximo de 1,8 ppm na sétima semana. A amônia, proveniente das excretas dos peixes, tanto fecais como branquiais, resultante do metabolismo proteico e também da ração não consumida (Tyson et al., 2008) deve ser nitrificada pelo processo denominado nitrificação para ser reduzida a nitrato que será absorvido pelos vegetais do sistema. 
Segundo Pereira \& Mercante (2005), a formação de compostos nitrogenados reduzidos, como, por exemplo, a amônia, ocorre como resultado da decomposição aeróbia e anaeróbia da matéria orgânica. A oxidação biológica desses compostos a nitrato é denominada nitrificação. A nitrificação é, na realidade, um processo que se caracteriza pela utilização de compostos inorgânicos reduzidos, por exemplo, o íon amônio, como doadores de hidrogênio, sendo que, pela sua oxidação, os microrganismos obtêm os equivalentes de redução para o processo de síntese.

A transformação da amônia em nitrato e nitrito é de fundamental importância para a manutenção do sistema de aquaponia (Figura 1).

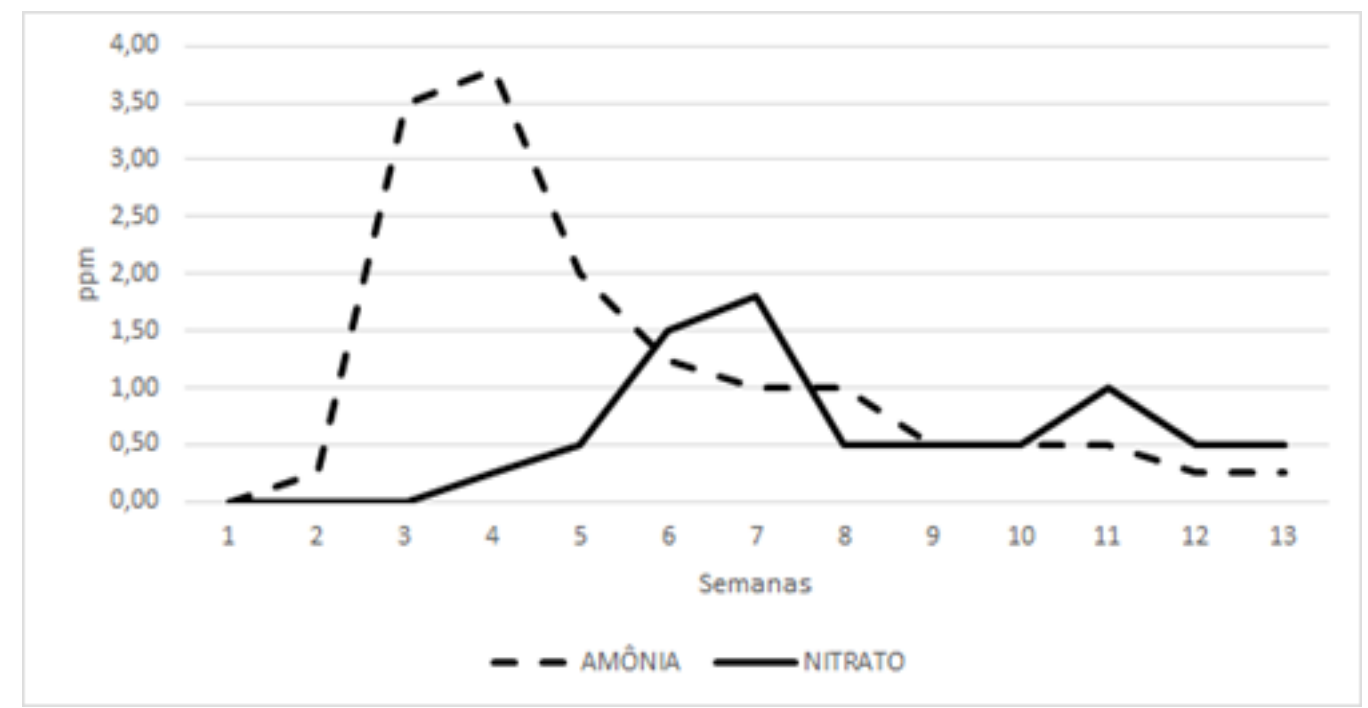

Figura 2. Níveis de amônia e nitrato mensurados durante o período de criação.

A produtividade de peixes no sistema de aquaponia relatada na literatura varia muito e é influenciada pelos componentes que compõem o sistema, como tipo de hidroponia utilizada, tipos de filtros biológicos e tanques de decantação, espécie de peixes, tipo de vegetais produzidos e a relação $\mathrm{m}^{2} \mathrm{de}$ hidroponia $/ \mathrm{m}^{3}$ de tanque de peixes (Goddek et al., 2016; Junge et al., 2017; Tyson et al., 2008; Yildiz et al., 2017).

Jordan et al. (2018) avaliando o rendimento de alfaces em diferentes substratos cultivadas em sistema de aquaponia trabalharam com 100 tilápias de aproximadamente $142 \mathrm{~g}$ por caixa de $1.000 \mathrm{~L}$. Densidade semelhante foi indicada por Coelho et al. (2014) que analisaram o desempenho zootécnico de tilápias em diferentes densidades de estocagem em sistema de recirculação de água com a microalga Spirulina platensis, concluindo que 0,1 alevinos de aproximadamente $10 \mathrm{~g}$ por litro apresentaram melhor crescimento.

O desempenho produtivo durante os três meses de acompanhamento foi considerado satisfatório (Tabela1).

Tabela 1. Desempenho produtivo de tilápias em sistema de aquaponia

\begin{tabular}{lc}
\hline Parâmetros de desempenho & 88,88 \\
\hline Sobrevivência (\%) & 50 \\
Peso médio inicial (g/peixe) & 1.500 \\
Biomassa inicial (g/tanque) & 352 \\
Peso médio final (g/peixe) & 9.856 \\
Biomassa inicial (g/tanque) & 3,36 \\
Ganho de peso médio diário (g/dia) & 945 \\
Consumo médio de ração (g/peixe) & 2,68 \\
Conversão alimentar aparente & \\
\hline
\end{tabular}

A mortalidade observada foi de 2, 3 e 5 peixes no sistema 1, 2 e 3 respectivamente, o que representou $11,1 \%$, consequentemente $88,9 \%$ de sobrevivência. Em sistema de recirculação Coelho et al. (2014) 
descrevem sobrevivência semelhante ao trabalharem com alevinos de tilápias. Em sistemas convencionais e produções comerciais o esperado é uma sobrevivência superior a $90 \%$, e em produção onde não haja a ação de predadores naturais como pássaros, a tendência é que a sobrevivência ultrapasse os $95 \%$. Neste trabalho, a mortalidade acentuada foi observada nos primeiros 15 dias do cultivo, o que pode ser atribuído à falta de maturidade do sistema de filtragem biológica e ao alto nível de amônia na primeira semana.

O ganho de peso médio diário observado foi de 3,36g, atendendo as expectativas de crescimento e ganho de peso apontadas por Somerville et al. (2014) que revelam que em condições ideais, as tilápias podem crescer de um peixe com $50 \mathrm{~g}$ até a sua maturidade $(500 \mathrm{~g})$ em cerca de seis meses. Ganho semelhante foi descrito por Mesquita et al. (2016) trabalhando com sistema de recirculação e tilápias. Esse ganho pode ser considerado satisfatório para sistema de produção intensivo e pode ser atribuída a temperatura constante do sistema e ao fato de estar em estufa agrícola. Os valores obtidos nas biometrias demonstraram crescimentos finais significativos dos peixes no período avaliado. O peso médio de $352 \mathrm{~g}$ está próximo do padrão comercial para venda de peixe inteiro e do filé (Massago et al., 2010). Os autores apontam que para tilápias o peso superior a $400 \mathrm{~g}$ é o mais indicado para abate, pois os rendimentos obtidos para as partes comestíveis são superiores. Comparando os dados do presente trabalho aos de outros com cultivo de tilápia em sistemas aquaponicos (Coelho et al., 2014; Hundley \& Navarro, 2013), verifica-se que os resultados foram superiores ao esperado, no período de 90 dias. Para atingir o peso médio de $352 \mathrm{~g}$, as tilápias apresentaram um consumo de $945 \mathrm{~g}$ de ração. A partir destes resultados foi calculada a conversão alimentar aparente, que relaciona o consumo de ração e o ganho de peso, assim quanto, pior a conversão alimentar, maior o gasto com ração. Entretanto a conversão alimentar pode variar de acordo com o sistema de produção. Além disso, segundo Kubitza (1999), a conversão alimentar pode ser afetada pela densidade de estocagem, pela idade e pelo tamanho dos peixes, sendo que as melhores conversões alimentares geralmente se encontram na faixa de 1,4 a 1,8. A conversão de 2,68 observada nesse sistema pode ser considerada alta e, provavelmente se deve ao manejo alimentar composto de apenas duas alimentações diárias.

\section{Conclusão}

A qualidade de água, após alguns dias de funcionamento do sistema foi se estabilizando e se manteve dentro dos parâmetros de qualidade exigidos. A integração da produção de tilápias com o cultivo de alfaces se mostrou eficiente na manutenção da qualidade de água, e o sistema em escadas proporcionou oxigenação suficiente para os peixes dispensando a necessidade de aeradores, até a biomassa observada.

Apesar do sistema ainda estar em expansão no Brasil, a modalidade tende a crescer muito nos próximos anos, tendo em vista que a aquaponia é um sistema extremamente útil e uma possível solução para os problemas hídricos e a falta de solos férteis, o sistema se torna uma alternativa viável e vantajosa, para uma produção de larga escala.

\section{Referências bibliográficas}

Boyd, C. E. (2000). Water quality: an introduction: Springer Science \& Business Media.

Castagnolli, N. (1992). Piscicultura de água doce. Jaboticabal, São Paulo: FUNEP.

Castillo-Castellanos, D., Zavala-Leal, I., Ruiz-Velazco, J. M. J., Radilla-García, A., Nieto-Navarro, J. T., Romero-Bañuelos, C. A. \& González-Hernández, J. (2016). Implementation of an experimental nutrient film technique-type aquaponic system. Aquaculture International, 24(2):637-646.

Coelho, A. A. d. C., Bezerra, J. H. C., Silva, J. W. A., Moreira, R. T., Albuquerque, L. F. G. \& Farias, W. R. L. (2014). Desempenho zootécnico de alevinos de tilápia do Nilo cultivados em um sistema de recirculação de água com a microalga" Spirulina platensis". Revista Brasileira de Saúde e Produção Animal, 15(1):149-159.

Crepaldi, D. V., Teixeira, E. A., Faria, P. M. C., Ribeiro, L. P., Melo, D. C., Carvalho, D., .. Saturnino, H. M. (2006). Sistemas de produção na piscicultura. Revista Brasileira de Reprodução Animal, 30(34):86-99.

Danaher, J. J., Shultz, R. C., Rakocy, J. E. \& Bailey, D. S. (2013). Alternative solids removal for warm water recirculating raft aquaponic systems. Journal of the World Aquaculture Society, 44(3):374383. 
Domingues, D. S., Takahashi, H. W., Camara, C. A. P. \& Nixdorf, S. L. (2012). Automated system developed to control $\mathrm{pH}$ and concentration of nutrient solution evaluated in hydroponic lettuce production. Computers and Electronics in Agriculture, 8453-61.

El-Sayed, A. M. (2006). Tilapia culture. London, U.K.: Cambridge University.

Eler, M. N. \& Millani, T. J. (2007). Métodos de estudos de sustentabilidade aplicados a aquicultura. Revista Brasileira de Zootecnia, 3633-44.

Garcia, L. d. O., Copatti, C. E., Wachholz, F., Pereira Filho, W. \& Baldisserotto, B. (2008). Freshwater temperature in the state of Rio Grande do Sul, Southern Brazil, and its implication for fish culture. Neotropical Ichthyology, 6(2):275-281.

Goddek, S., Schmautz, Z., Scott, B., Delaide, B., Keesman, K. J., Wuertz, S. \& Junge, R. (2016). The effect of anaerobic and aerobic fish sludge supernatant on hydroponic lettuce. Agronomy, 6(2):1-12.

Hundley, G. C. \& Navarro, R. D. (2013). Aquaponia: a integração entre piscicultura e a hidroponia. Revista Brasileira de Agropecuária Sustentável, 352-61.

IBGE, (2019). Pesquisa Pecuária Municipal: Produção da aquicultura. Disponível a partir de: http://www.sidra.ibge.gov.br/bda/tabela/listabl.asp?c=3940\&z=t\&o=21, Data de acesso: 28 de outubro de 2019.

Jordan, R. A., Geisenhoff, L. O., Oliveira, F. C., Santos, R. C. \& Martins, E. A. S. (2018). Yield of lettuce grown in aquaponic system using different substrates. Revista Brasileira de Engenharia Agrícola e Ambiental, 22(1):27-31.

Junge, R., König, B., Villarroel, M., Komives, T. \& Jijakli, M. H. (2017). Strategic points in aquaponics. Water, 1821-9.

Kloas, W., Groß, R., Baganz, D., Graupner, J., Monsees, H., Schmidt, U., . . Wittstock, B. (2015). A new concept for aquaponic systems to improve sustainability, increase productivity, and reduce environmental impacts. Aquaculture Environment Interactions, 7(2):179-192.

Kubitza, F. (1999). Nutrição e alimentação dos peixes cultivados (Vol. 1). Campo Grande, Mato Grosso do Sul.

Kubitza, F. (2006). Sistemas de Recirculação: Sistemas fechados com tratamento e reuso da água. Panorama da Aqüicultura, 16(95):15-22.

Love, D. C., Fry, J. P., Genello, L., Hill, E. S., Frederick, J. A., Li, X. \& Semmens, K. (2014). An international survey of aquaponics practitioners. PloS One, 9(7):e102662.

Massago, H., Castagnolli, N., Malheiros, E. B., Koberstein, T. C. R. D., Santos, M. A. \& Ribeiro, R. P. (2010). Crescimento de quatro linhagens de tilápia Oreochromis niloticus. Revista Acadêmica Ciência Animal, 8(4):397-403.

Maucieri, C., Nicoletto, C., Junge, R., Schmautz, Z., Sambo, P. \& Borin, M. (2018). Hydroponic systems and water management in aquaponics: a review. Italian Journal of Agronomy, 1310-12.

Mercante, C. T. J., Cabianca, M., Silva, D., Costa, S. V. \& Esteves, K. E. (2004). Water quality in feefishing ponds located in the metropolitan region of São Paulo city, Brazil: an analysis of the eutrophication process. Acta Limnologica Brasiliensia, 16(1):95-102.

Mesquita, R. C. T., Maschio, D., Eloy, L., Godoy, L., Paz, É. M. \& Streit Júnior, D. P. (2016). Vantagens do cultivo de tilápia do Nilo (Oreochromis niloticus) com mínima liberação de efluentes. Revista Brasileira de Higiene e Sanidade Animal, 10(3):447-454.

Peixe Br. Anuário Peixe BR da Piscicultura (2019). Associação Brasileira de Piscicultura, São Paulo, SP, Brasil.

Pereira, L. P. F. \& Mercante, C. T. J. (2005). A amônia nos sistemas de criação de peixes e seus efeitos sobre a qualidade da água. Uma revisão. Boletim do Instituto de Pesca, 31(1):81-88.

Rakocy, J. (2007). Ten guidelines for aquaponic systems. Aquaponics Journal, 4614-17.

Rakocy, J., Masser, M. P. \& Losordo, T. (2016). Recirculating aquaculture tank production systems: aquaponics-integrating fish and plant culture. Southern Regional Aquaculture Center Publications, 4541-16. 
Rakocy, J. E. (2012). Aquaponics: integrating fish and plant culture. Aquaculture Production Systems, 1344-386.

Sátiro, T. M., Neto, K. X. C. R. \& Delprete, S. E. (2018). Aquaponia: Sistema que integra produção de peixes com produção de vegetais de forma sustentável. Revista Brasileira de Engenharia de Pesca, 11(1):38-54.

Somerville, C., Cohen, M., Pantanella, E., Stankus, A. \& Lovatelli, A. (2014). Small-scale aquaponic food production: integrated fish and plant farming. FAO Fisheries and Aquaculture Technical Paper, 1(589):I.

Souza, E. C. P. M. \& Teixeira Filho, A. R. (1985). Piscicultura fundamental. São Paulo: Nobel.

Tyson, R. V., Simonne, E. H. \& Treadwell, D. D. (2008). Reconciling pH for ammonia biofiltration in a cucumber/tilapia aquaponics system using a perlite medium. Hort Science, 43, 719-724., 43719724.

Yildiz, H. Y., Robaina, L., Pirhonen, J., Mente, E., Domínguez, D. \& Parisi, G. (2017). Fish welfare in aquaponic systems: its relation to water quality with an emphasis on feed and faeces-a review. Water, 9(13):1-17.

Recebido: 13 de novembro, 2019.

Aprovado: 11 de dezembro, 2019.

Publicado: 29 de fevereiro, 2020.

Licenciamento: Este artigo é publicado na modalidade Acesso Aberto sob a licença Creative Commons Atribuição 4.0 (CC-BY 4.0), a qual permite uso irrestrito, distribuição, reprodução em qualquer meio, desde que o autor e a fonte sejam devidamente creditados. 